\title{
Uso popular de plantas medicinais na promoção da saúde animal em assentamentos rurais de Seropédica $-\mathbf{R} \mathbf{J}^{*}$
}

\section{Popular use of medicinal plants promoting animal health in rural settlements in Seropédica - RJ}

\author{
Welverson Marlon O. da Silva, ${ }^{* *}$ Gisele Fátima X. T. de Souza, ${ }^{* *}$ Pollyanna Barbosa Vieira , ${ }^{* *}$ Argemiro Sanavria**
}

\begin{abstract}
Resumo
O emprego da fitoterapia esteve presente ao longo da história, sendo utilizada no tratamento das enfermidades que acometem o ser humano. Tal afirmativa também é válida para a saúde animal, principalmente em comunidades rurais. Este projeto objetivou resgatar o conhecimento popular sobre o uso de plantas medicinais em animais nas comunidades do Assentamento Rural Sol da Manhã e do Mutirão Eldorado, situadas no município de Seropédica. Foram realizadas visitas aos assentamentos, aplicando-se questionários sobre indicações terapêuticas e formas de utilização das espécies no tratamento de animais domésticos. Exemplares das espécies utilizadas foram identificados pelo Departamento de Botânica da Universidade Federal Rural do Rio de Janeiro (UFRRJ). No total foram entrevistadas 52 famílias, das quais $63,5 \%$ afirmaram utilizar plantas medicinais no tratamento de saúde dos seus animais. Dentre as 30 espécies medicinais citadas foram evidenciadas 12 indicações gerais, das quais três destacaramse: anti-helmíntica (36,58\%), anti-inflamatória (17,07\%), e repelente (12,19\%), caracterizando a importância do emprego das plantas no tratamento de doenças parasitárias, sendo a principal espécie indicada para esse uso o Chenopodium ambrosioides L. (erva-de-santa-maria) com $25 \%$ das citações. A correlação entre o conhecimento popular e as pesquisas científicas pode gerar novas intervenções terapêuticas para as diversas doenças que acometem animais.
\end{abstract}

Palavras-chave: fitoterapia, conhecimento popular, comunidades rurais.

\begin{abstract}
The employment of phytotherapy has been present throughout history, being utilized in the treatment of infirmities that affect the human being. Such statement is also valid for animal health, mainly in rural communities. This project aimed to rescue the popular knowledge about the use of medicinal plants in animals, by the communities of Sol da Manhã Rural Settlement and Mutirão Eldorado, located in Seropédica. Visits to the settlements were performed, and interviews submitted about the therapeutic indications and methods of utilization of species in the treatment of domestic animals. Specimens of the utilized species were identified by the Botanic Department of Universidade Federal Rural do Rio de Janeiro (UFRRJ). 52 families were interviewed, amongst which $63,5 \%$ claimed to use medicinal plants on the treatment of their animals. Out of 30 medicinal species mentioned, 12 general indications have been evidenced, of which three were highlighted: anthelmintic (36,58\%), anti-inflammatory (17,07\%), and repellent $(12,19 \%)$, characterizing the importance of the employment of plants for the treatment of parasitic diseases, being the Chenopodium ambrosioides L. (herba sancti mariæ) the most indicated species for this use, with $25 \%$ of the quotations. The correlation between popular knowledge and scientific research may generate new therapeutic interventions for the various animal diseases.
\end{abstract}

Keywords: phytotherapy, popular knowledge, rural communities.

\section{Introdução}

O uso das plantas medicinais empregadas tanto para a alimentação da população humana quanto para fins medicinais, esteve presente ao longo do tempo (Almassy Junior et al., 2004). Esta opção terapêutica é a alternativa de muitos brasileiros, principalmente em regiões com infraestrutura deficitária (Lima et al., 2007), utilizando-se dessa prática para si e para seus animais.

O conhecimento tradicional do uso das espécies medicinais é repassado, principalmente, de forma empírica entre indivíduos de uma mesma comunidade, sendo mantido por meio da tradição oral (Oliveira et al., 2009). Porém, devido à necessidade de validação dos vários usos terapêuticos e consequentemente dos efeitos benéficos e maléficos causados pelo uso de plantas medicinais, nem sempre é aceita a forma de utilização popular (Oliveira et al., 2007).

O resgate e a transmissão de conhecimentos acerca do plantio e uso adequado das espécies medicinais são de grande importância, pois muitas pessoas não sabem identificá-las, ou as preparam e as utilizam incorretamente, além de não conhecerem os riscos dos efeitos colaterais e tóxicos, colocando em risco a sua saúde e a de seus animais. Há de se ressaltar que muitas plantas úteis aos seres humanos podem produzir

\footnotetext{
*Recebido em 22 de novembro de 2012 - Aceito em 15 de março de 2013.

** Departamento de Epidemiologia e Saúde Pública da Universidade Federal Rural do Rio de Janeiro, BR 465, km 47, CEP 23890-000, Seropédica, RJ, Brasil.

Autor para correspondência: Welverson Marlon O. da Silva. E-mail: wmarlon_mvrural@yahoo.com.br.
} 
substâncias potencialmente tóxicas, se utilizadas em dosagens inadequadas (Oliveira et al., 2009). Carlini (2004) afirma que, dentre os principais problemas causados pelo uso indiscriminado e prolongado de espécies medicinais, estão as reações alérgicas e os efeitos tóxicos em vários órgãos. Portanto, é de grande importância conscientizar a população sobre o uso adequado das plantas e medicamentos naturais.

A ampliação dos cuidados e preocupações com os animais de companhia ou de produção está diretamente relacionada com a questão do bem-estar animal e a saúde de ambos. Para garantir a sanidade do animal, é necessário preservar o seu estado de saúde, evitando a transmissão de doenças a outros animais e até mesmo aos seres humanos (Porcher, 2004).

Na medicina veterinária, ressalta-se que a administração de subdosagens, a persistência da utilização de anti-helmínticos com o mesmo princípio ativo e o momento inadequado de everminação geram demanda por métodos alternativos de controle de helmintoses, dentre eles o uso de espécies medicinais (Rotondano et al., 2008). Segundo Vieira (2003), o uso da fitoterapia no controle de verminoses visa reduzir o custo com a aquisição de anti-helmínticos, além de prolongar o aparecimento de resistência por uso contínuo desses medicamentos.

Nesse contexto, este trabalho teve como objetivo principal realizar um levantamento da biodiversidade botânica utilizada com fins medicinais em animais, pelas comunidades do Assentamento Rural Sol da Manhã e do Mutirão Eldorado, situadas no município de Seropédica, assim como as principais indicações terapêuticas e formas de uso das espécies, promovendo o resgate do conhecimento tradicional.

\section{Material e métodos}

Foram realizadas visitas técnicas às propriedades do Assentamento Rural Sol da Manhã e do Mutirão Eldorado, com aplicação de 52 questionários elaborados com respostas abertas e fechadas, adotando-se metodologia proposta por Amaro et al. (2004). Abordou-se o uso de plantas com conduta terapêutica para diferentes enfermidades em animais domésticos, sua forma de utilização (parte utilizada da planta e modo de administração) e sua indicação terapêutica, além das principais espécies animais presentes nos assentamentos.

Durante as entrevistas, foram obtidos 30 exemplares de espécies de plantas utilizadas pelas comunidades, os quais foram devidamente armazenados, identificados e alocados em exsicatas pelo Departamento de Botânica do Instituto de Biologia da Universidade Federal Rural do Rio de Janeiro (UFRRJ).

No decorrer do projeto, foram distribuídos 100 folders e 70 cartilhas às comunidades assistidas. Os informativos abordaram a importância do uso adequado das plantas medicinais (identificação, coleta, secagem, conservação, modo de preparo e indicação), a fim de evitar possíveis agravos e efeitos tóxicos.

\section{Resultados e discussão}

A partir dos dados obtidos nas entrevistas, observou-se que $63,5 \%$ das famílias afirmaram utilizar plantas medicinais como forma terapêutica nas diversas afecções dos animais domésticos, caracterizando a relevância dessa prática nestas comunidades rurais.
Verificou-se que $82,7 \%$ dos moradores possuíam aves e cães, sendo estas as espécies animais predominantes. Essa relação íntima entre humanos e animais deve ser vista com olhar criterioso, devido à possibilidade de ocorrência de zoonoses, principalmente em comunidades rurais onde o status sanitário, geralmente, é baixo. Corroborando com Leite et al. (2007), que explicitam a importância do estudo epidemiológico das doenças de pequenos e grandes animais, sendo essencial em saúde pública e clínica veterinária, já que muitos gêneros de helmintos e protozoários com importante potencial zoonótico utilizam-se dos animais domésticos como hospedeiros e podem contaminar facilmente o homem.

As espécies vegetais citadas foram identificadas e listadas com sua respectiva: indicação terapêutica, via de administração, espécie animal a ser tratada, parte utilizada e modo de preparo, de acordo com as informações fornecidas pelas comunidades rurais (Tabela 1 ).

Dentre as 12 indicações gerais citadas, três se destacaram: anti-helmíntica $(36,58 \%)$, anti-inflamatória $(17,07 \%)$, e repelente (12,19\%), demonstrando a importância das plantas com ação antiparasitária, principalmente para comunidades onde o medicamento convencional é de difícil aquisição. As infecções por parasitos representam um problema de saúde pública no Brasil, sendo sua alta incidência devido às precárias condições de saneamento básico, habitação e educação (Belizário e Silva, 2012). Este resultado se apresenta como indicativo da saúde animal e alarme para a saúde humana nos casos onde existam poucos cuidados com a higiene.

Foram identificadas 30 espécies medicinais, sendo quatro destas mais citadas pelas famílias entrevistadas: Chenopodium ambrosioides L. (erva-de-santa-maria) citado por $25 \%$, Citrus $s p$ (limão) citado por 25\%, Allium sativum L. (alho) citado por $18,75 \%$ e Gallesia integrifolia (Spreng.) Harms (pau-d'alho) citado por $9,37 \%$.

O Chenopodium ambrosioides L. foi a principal espécie indicada como vermífugo, sendo empregada comumente no tratamento de helmintoses de cães, revelando-se como medida alternativa de baixo custo ou medida complementar, associada a outras formas de tratamento. Esta espécie está relacionada nos levantamentos da Organização Mundial da Saúde como uma das plantas mais utilizadas dentre os remédios naturais do mundo inteiro (Lorenzi e Matos, 2002). O ascaridol (princípio ativo vermífugo da planta) é encontrado nas folhas e nos frutos da erva-de-santa-maria.

O Citrus sp (limão) foi indicado para o tratamento das enfermidades respiratórias de galináceos, sendo administrado o suco do fruto, similar ao observado por Alves et al. (2009) e Andrade et al. (2012) onde o suco do limão foi utilizado por comunidades rurais no tratamento de doenças respiratórias que acometem aves, demonstrando o comum emprego desta prática por esse grupo populacional. Embora a indicação do limão no tratamento de resfriados seja geralmente associada à vitamina $\mathrm{C}$, há diversas discussões quanto à comprovada eficácia dela para este fim. Segundo Trucom (2004), a vitamina C favorece a defesa do organismo, sendo um antioxidante poderoso que exerce papel inestimável nos fenômenos oxidorredutores, portanto, um protetor aos danos do envelhecimento (oxidação) de todas as células e tecidos, beneficiando o rejuvenescimento e o desempenho das glândulas endócrinas. 
Tabela 1: Espécies vegetais citadas, com fins terapêuticos em animais, por famílias do Assentamento Rural Sol da Manhã e do Mutirão Eldorado: família/ nome científico/ nome vulgar, parte utilizada, indicação terapêutica, espécie animal, modo de preparo e via de administração

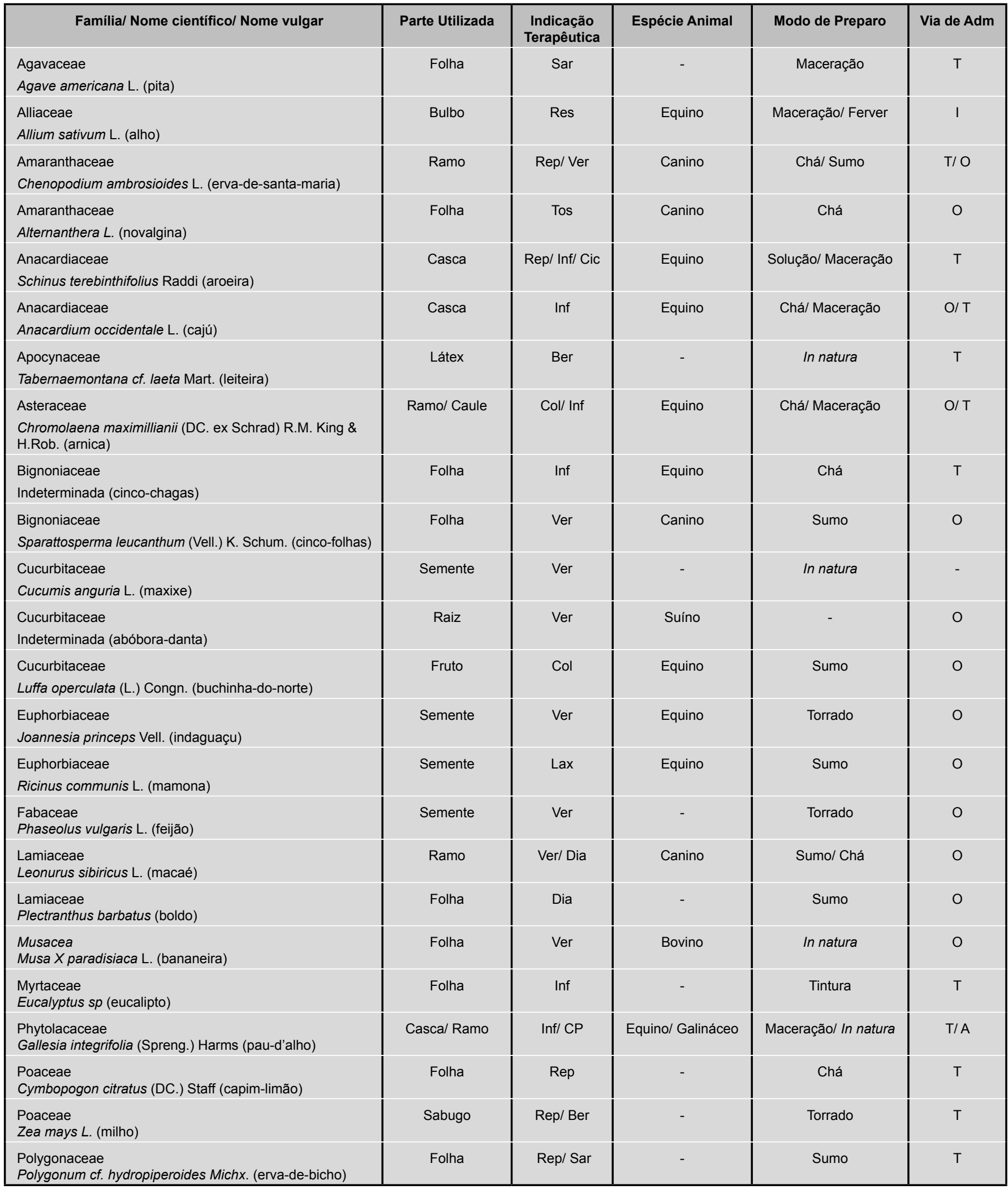




\begin{tabular}{|l|c|c|c|c|}
\hline $\begin{array}{l}\text { Rubiaceae } \\
\text { Coffea Arabica L. (café) }\end{array}$ & Fruto & Col & Equino & Maceração \\
$\begin{array}{l}\text { Rutaceae } \\
\text { Citrus sp (limão) }\end{array}$ & Fruto & Res & Galináceo & Suco \\
$\begin{array}{l}\text { Rutaceae } \\
\text { Ruta graveolens L. (arruda) }\end{array}$ & Folha & Ver & - & Sumo \\
$\begin{array}{l}\text { Solanaceae } \\
\text { Indeterminada (tomate-miudo) }\end{array}$ & Folha & Dia & - & Chá \\
$\begin{array}{l}\text { Solanaceae } \\
\text { Nicotiana tabacum L. (fumo-de-rolo) }\end{array}$ & Folha \\
$\begin{array}{l}\text { Solanaceae } \\
\text { Solanum sp (laranja-do-mato) }\end{array}$ & Folha & Dia & Aquecer \\
em óleo & Chá \\
\hline
\end{tabular}

Sar=sarna; Rep=repelente; Tos=tosse; Res=resfriado; Ver=vermífugo; Inf=Inflamação; Cic=cicatrizante; Ber=berne; Col=cólica; Lax=laxante; Dia=diarréia; $\mathrm{CP}=$ carrapato e pulga. $\mathrm{T}=$ tópico; $\mathrm{O}=$ oral; I=inalatório; $\mathrm{A}=$ =ambiente.

O Allium sativum L. foi indicado no tratamento de afecções respiratórias de equinos, através da inalação do vapor do bulbo macerado e fervido em água. Segundo Marchiori (2005), a maior concentração de fitoquímicos terapêuticos do alho encontra-se nos bulbos, popularmente conhecidos como dentes de alho, sendo a alicina e o ajoeno os principais compostos responsáveis pela ação anti-inflamatória, vasodilatadora, hipotensora, antibiótica, antifúngica e antiviral, caracterizando a espécie como possuidora de diversos usos terapêuticos.

A Gallesia integrifolia (Spreng.) Harms (pau-d'alho) foi indicada pelas comunidades para o tratamento de inflamações em equinos, utilizando o macerado da casca, além de ser usada no controle de carrapatos e pulgas de galináceos no ambiente. Segundo Fraga et al. (2006), o óleo essencial de G. integrifolia (Sprengel) Harms apresenta teores de substâncias conhecidamente antimicrobianas, demonstrando a importância do levantamento das espécies do gênero Gallesia.

Ao serem questionados sobre ocorrência de efeitos adversos das espécies medicinais, os moradores informaram que nunca os observaram. Caso tenham ocorrido, não foram percebidos, talvez por necessitar de uso prolongado ou por apresentarem efeitos brandos. Relato que corrobora com o de Souza (1991) ao tratar ratos por via oral e não detectar efeitos colaterais, apesar da toxicidade do ascaridol. Sabe-se também que o uso de fitoterápicos tanto na medicina humana como na veterinária deve-se a algumas vantagens. Dentre elas destacam-se: preço reduzido e menores efeitos colaterais, quando comparados aos medicamentos convencionais (Marinho et al., 2007).

\section{Conclusão}

O levantamento da biodiversidade botânica utilizada no tratamento de animais, em comunidades rurais, demonstra o comum emprego desta prática nessas localidades, principalmente devido ao fácil acesso e ao baixo custo, além de possibilitar o resgate do conhecimento popular sobre as indicações terapêuticas e formas de utilização das espécies vegetais. Porém esse conhecimento nem sempre é comprovado, devendo ser correlacionado ao técnico-científico, a fim de possibilitar novas intervenções terapêuticas, promover a saúde e o bem-estar dos animais e evitar a ocorrência de possíveis agravos à saúde humana.

\section{Agradecimentos}

À equipe coordenadora do Programa de Bolsas Institucionais de Extensão da UFRRJ, aos técnicos do Laboratório de Parasitologia do Instituto de Veterinária da UFRRJ e aos profissionais do Departamento de Botânica do Instituto de Biologia da UFRRJ.

\section{Referências}

ALMASSY JUNIOR, A. A. Análise das características etnobotânicas e etnofarmacológicas de plantas medicinais na comunidade de Lavras Novas, ouro Preto/MG. [Doutorado]. Universidade Federal de Viçosa, 2004. xiv. $132 \mathrm{f}$. il $29 \mathrm{~cm}$.

ALVES, B. C.; SOUZA, R. M. ; CUNHA, R. F. A.; PINTO, J. G. P.; OLIVEIRA, F. G. Diagnóstico sanitário na criação de pequenos animais em comunidades rurais do agreste paraibano. Simpósio Paraibano de Meio Ambiente, Cajazeiras - PB, set. 2009.

AMARO, A.; PÓVOA A.; MACEDO, L. Faculdade de Ciências da Universidade do Porto - Departamento de Química. A arte de fazer questionários. Metodologias de Investigação em Educação, 2004. ANDRADE, S. E. O.; MARACAJÁ, P. B.; SILVA, R. A.; FREIRES, G. F.; PEREIRA, A. M. Estudo etnoveterinário de plantas medicinais na comunidade Várzea Comprida dos Oliveiras, Pombal, Paraíba, Brasil. Revista Verde de Agroecologia e Desenvolvimento Sustentável. Artigo Científico, Mossoró - RN, v. 7, n. 2, p. 193-198, 2012.
BELIZÁRIO, T. L.; SILVA, L. A. abordagem etnobotância no tratamento de (confirmar) parasitoses em comécios de fitoterápicos e numa comunidade rural em Uberlândia-MG. Enciclopédia Biosfera, Goiânia, v. 8, n. 15, 2012.

CARLINI, E. Entre conhecimento popular e científico. 2004. Disponível em <http.www.comciencia.br>. Acesso em 31/jan./2012. FRAGA, H. F.; ROSA, P. M.; MORAIS, A. A.; PINTO, A. C.; REZENDE, C. M.

Análise dos constituintes químicos do óleo essencial das folhas de Gallesia integrifolia (Sprengel) Harms (Phytolaccaceae). XXIX Reunião Anual da Sociedade Brasileira de Química, Águas de Lindóia - SP, maio 2006.

LEITE, L.C.; CÍRIO, S.M.; NAVARRO-SILVA, M.A.; ZADOROSNEI, A.C.B.; LUZ, E.; MARINONI, L.P.; LEITE, S.C.; LUNELLI, D. Ocorrência de endoparasitas em amostras de fezes de cães (Canis familiaris) da região metropolitana de Curitiba, ParanáBrasil. Estud. Biol. n. 29 (67/69), p. 319-326, 2007. 
LIMA, S. G. V.; LIMA, A. F.; DONAZZOLO, J. L. Resgate do conhecimento popular e uso de plantas medicinais na promoção da saúde em Sananduva - RS. Rev. Bras. Agroecologia, v. 2, n. 1, fev. 2007.

Lorenzi, H. \& Matos, F. J. A. Plantas Medicinais no Brasil - Nativas e Exóticas. São Paulo: Nova Odessa, 543 p., 2002.

MARCHIORI, V. F. Propriedades funcionais do alho (Allium sativum L.). ESALQ/USP - SP. 2005. Disponível em <http://www.esalq.usp. $\mathrm{br} / \mathrm{siesalq} / \mathrm{pm} / \mathrm{alho}$ revisado.pdf>. Acesso em 10/mar./2013.

MARINHO, M.L. A utilização de plantas medicinais em medicina veterinária: um resgate do saber popular. Revista Brasileira de Plantas Medicinais, Botucatu, v. 9, n. 3, p. 64-69, 2007.

OLIVEIRA, C. J.; ARAÚJO, T. L. Plantas medicinais: usos e crenças de idosos portadores de hipertensão arterial. Revista Eletrônica de Enfermagem, v. 9, n. 1, p. 93-105, 2007.

OLIVEIRA, L. S. T.; CUNHA E SILVA, S. L.; TAVARES, D. C.; SANTOS, A.V.S.; OLIVEIRA, G. C. B. Uso de plantas medicinais no tratamento de animais. Enciclopédia Biosfera, Goiânia, v. 5, n. 8, 2009.
PORCHER, J. "Você liga demais para os sentimentos" "Bem-estar animal", repressão da afetividade, sofrimento dos pecuaristas. Produção, São Paulo - SP, v. 14, n. 3, 2004.

ROTONDANO, T. E. F.; SOUZA, G. A. F.; LIMA, F. H.; FILHO, J. M. P.; RODRIGUES, O. G.; ATHAYDE, A. C. R. Difusão do uso de plantas medicinais com ação antiparasitária na produção de caprinos do sistema de produção da Região de Patos - PB. III Encontro de Extensão da UFCG, nov. 2008.

SOUZA, M.P.; MATOS M.E.O.; MATOS F.J.A. ET AL. Constituintes químicos de plantas medicinais brasileiras. Fortaleza: Impr. Universitária/UFC, 416 p. 1991.

TRUCOM, C. O poder de cura do limão - um guia de medicina caseira. Ed. Alaúde. 200 p., 2004.

VIEIRA, L.S. Alternativa de controle da verminose gastrintestinal dos pequenos ruminantes, Ministério de agricultura pecuária e abastecimento - circular técnica 29, Sobral - CE, 2003. Disponível em <http://www.sheepembryo.com.br/files/artigos/119.pdf>. Acesso em 29/jan./2012. 